\section{Zika Virus Detection with 2013 Serosurvey, Mombasa, Kenya}

\author{
Elizabeth Hunsperger, Dennis Odhiambo, \\ Albina Makio, Moshe Alando, Melvin Ochieng, \\ Victor Omballa, Peninah Munyua, Godfrey Bigogo, \\ M. Kariuki Njenga, Marc-Alain Widdowson \\ Author affiliations: US Centers for Disease Control and \\ Prevention, Nairobi, Kenya (E. Hunsperger, P. Munyua, \\ M.-A. Widdowson); Kenya Medical Research Institute, Nairobi, \\ Kenya (D. Odhiambo, A. Makio, M. Alando, M. Ochieng, \\ V. Omballa, G. Bigogo); Washington State University, Pullman, \\ Washington, USA (M.K. Njenga); Institute of Tropical Medicine, \\ Antwerp, Belgium (M.-A. Widdowson)
}

DOI: https://doi.org/10.3201/eid2607.191363

Acute Zika virus (ZIKV) infection has not been confirmed in Kenya. In 2018, we used specimens collected in a 2013 dengue serosurvey study in Mombasa to test for ZIKV IgM. We confirmed specific ZIKV IgM positivity in 5 persons. These results suggest recent ZIKV transmission in the coastal region of Kenya.

7 ika ika virus (ZIKV), an arbovirus belonging to the family Flaviviridae, was initially isolated in 1947 from the serum of a pyrexic sentinel rhesus macaque in the canopy of the Zika Forest in Uganda (1). In 1970, ZIKV antibodies were reported in humans in Kenya, using immunoassays with limited sensitivity and specificity (2); since then, only past infections have been reported $(3,4)$.

In 2015, the emergence of ZIKV in the Americas (5) raised the possibility that ZIKV infections were also occurring in Kenya but were undetected due to mild or asymptomatic infections and rudimentary birth defect surveillance. In 2016, Cape Verde and, in 2017, Angola reported ZIKV outbreaks associated with microcephaly. Genome sequencing from Angola identified the similarity of the Asian lineage of ZIKV to the American strain (6); this evidence implies that the African lineage may be less likely to cause microcephaly, thus explaining the few reported number of ZIKV-associated microcephaly cases in Africa (7).

To understand prior transmission of ZIKV in Kenya, in 2018, we used specimens collected in 2013 for a dengue serosurvey from Mombasa amidst a dengue outbreak. During May 3-11, 2013, serum specimens were collected from 1,500 consenting household members in 986 randomly selected households (8). Specimens were tested for dengue virus (DENV) by real-time reverse transcription PCR (rRT-PCR) and DENV IgM ELISA (InBios International, Inc., https:/ / inbios.com). Concurrent with this serosurvey, hospital surveillance of suspected dengue patients was established (8).

For our study, we used only the DENV-negative serum specimens and tested them for ZIKV by Centers for Disease Control and Prevention rRT-PCR and ZIKV IgM antibody capture ELISA (MAC ELISA). A ZIKV MAC ELISA specimen with a positive-to-negative ratio $\geq 3.00$ was positive and a ratio of $2-2.99$ was equivocal. We confirmed all positive specimens with $90 \%$ plaque reduction neutralization test $\left(\mathrm{PRNT}_{90}\right)$ against ZIKV strain MR766 (African lineage) and DENV (ChimeraVax; Sanofi Pasteur, https://www. sanofi.com) (9). We defined recent ZIKV infection by a positive result in the ZIKV MAC ELISA and a PRNT $_{90}$ titer of ZIKV that was 4-fold higher than the titer of DENV. The institutional review board at US Centers for Disease Control and Prevention and Kenya Medical Research Institute Scientific Ethics Review Unit approved the study.

We identified a total of 745 DENV-negative persons from the dengue serosurvey $(\mathrm{n}=704)$ and hospital surveillance $(\mathrm{n}=41)$; median age was 28 (range 0-94) years. None were positive by ZIKV rRT-PCR. Thirty-four (4.6\%) were positive by ZIKV MAC ELISA, 7 captured in hospital surveillance; 24 (3.2\%) were equivocal and $687(92.2 \%)$ negative. Of the 34 ZIKV MAC ELISA positives, the ZIKV PRNT ${ }_{90}$ assay confirmed 5 (15.1\%, 4 from serosurvey and 1 hospital) as ZIKV, 7 (21.2\%) as DENV, 3 (9.1\%) as cross-reactive to both viruses, and $18(54.5 \%)$ as negative for ZIKV and DENV; 1 (2.9\%) could not be confirmed due to insufficient sample (Table). Of the 5 ZIKV MAC ELISA-positive patients confirmed by ZIKV PRNT ${ }_{90}$, median age was 57 (range 50-70) years.

We identified 5 persons sampled in 2013 who were positive for ZIKV IgM and confirmed by $\mathrm{PRNT}_{90}$. Of note, the positive participants (median age 57 years) were older than the sample group tested (median age 29 years). The level of transmission of ZIKV in Kenya is unknown, although Kenya has the competent vector (Aedes aegypti mosquitoes), and parts of Kenya are ecologically similar to the Zika forest (1). ZIKV has not been detected in Kenya despite recent intensive follow-up of pregnant women in coastal Kenya (10). Our study suggests that ZIKV may circulate in Mombasa and cause asymptomatic disease not captured in hospital surveillance systems. In addition, severely ill ZIKV patients might not have been identified from the original dengue study because it only captured clinical endpoints related to severe dengue. 
Table. Test results for 16 participants in a 2013 serosurvey in Kenya whose samples were positive for ZIKV by testing conducted in $2018^{*}$

\begin{tabular}{|c|c|c|c|c|c|c|}
\hline $\begin{array}{l}\text { Participant } \\
\text { no. }\end{array}$ & Age, y & $\begin{array}{c}\text { CDC ZIKV MAC } \\
\text { ELISA P/N ratio (IgM) }\end{array}$ & ZIKV PRNT $_{90}$ titer & DENV PRNT $_{90}$ titer & Participant type & $\begin{array}{c}\text { Final interpretation } \\
\text { of case }\end{array}$ \\
\hline 2 & 63 & 3.56 & $1: 20$ & $<1: 20$ & Serosurvey & ZIKV \\
\hline 3 & 50 & 10.00 & $>1: 320$ & $<1: 20$ & Serosurvey & ZIKV \\
\hline 5 & 70 & 3.17 & $1: 20$ & $<1: 20$ & Serosurvey & ZIKV \\
\hline 10 & NA & 3.51 & $1: 20$ & $<1: 20$ & Hospital & ZIKV \\
\hline 31 & 51 & 5.50 & $>1: 320$ & $1: 20$ & Serosurvey & ZIKV \\
\hline 4 & 45 & 3.26 & $<1: 20$ & $1: 20$ & Serosurvey & DENV \\
\hline 7 & 36 & 8.65 & $1: 20$ & $1: 320$ & Serosurvey & DENV \\
\hline 20 & 58 & 13.18 & $1: 20$ & $1: 320$ & Serosurvey & DENV \\
\hline 25 & 45 & 3.70 & $1: 20$ & $>1: 320$ & Serosurvey & DENV \\
\hline 26 & 27 & 5.00 & $1: 80$ & $>1: 320$ & Serosurvey & DENV \\
\hline 27 & 42 & 3.20 & $1: 80$ & $1: 320$ & Serosurvey & DENV \\
\hline 33 & 30 & 10.70 & $1: 20$ & $1: 80$ & Serosurvey & DENV \\
\hline 1 & 32 & 4.49 & $1: 20$ & $1: 20$ & Serosurvey & Cross-reactive \\
\hline 9 & 27 & 3.82 & $1: 20$ & $1: 20$ & Serosurvey & Cross-reactive \\
\hline 30 & 58 & 3.20 & $>1: 320$ & $>1: 320$ & Serosurvey & Cross-reactive \\
\hline 6 & 41 & 16.35 & $1: 20$ & ND & Serosurvey & Inconclusive \\
\hline
\end{tabular}

We found 1 participant positive by ZIKV MAC ELISA but negative by ZIKV and DENV PRNT ${ }_{90^{\prime}}$ suggesting the presence of another co-circulating cross-reactive flavivirus in this region of Kenya (e.g., West Nile virus). This finding merits further investigation to determine all circulating flaviviruses in Mombasa. ZIKV transmission season in Kenya most likely coincides with other arboviruses that share the same vector. Of interest, titers of neutralizing antibodies against ZIKV were low for 3 of the 5 positive participants (1:20 $\mathrm{PRNT}_{90}$ titer), typically observed in acute infections ( $<10$ days after onset of illness). Two participants had high neutralization titers (>1:320), suggesting recent infection (within the previous 90 days).

Our study had some limitations. It was conducted in 2018, so specimens had been archived for 5 years. We also had incomplete demographic and clinical data and could not discount concurrent DENV infections. Finally, we might have underestimated ZIKV positives because of weak neutralization by IgM.

In conclusion, ZIKV may have circulated at low levels in Kenya in 2013. More research is needed to evaluate current ZIKV circulation and characterize other co-circulating flaviviruses. Enhanced surveillance systems, including for microcephaly and other birth defects, could capture ZIKV patients and determine the epidemiology of ZIKV African lineage in this country.

This research was made possible through support provided by the Office of Infectious Disease, Bureau for Global Health, US Agency for International Development, under the terms of an Interagency Agreement with CDC.

\section{About the Author}

Dr. Hunsperger is a researcher with the US Centers for Disease Control and Prevention, Nairobi, Kenya. Her research interest is vectorborne diseases.

\section{References}

1. Dick GW, Kitchen SF, Haddow AJ. Zika virus. I. Isolations and serological specificity. Trans R Soc Trop Med Hyg. 1952;46:509-20. http://dx.doi.org/10.1016/ 0035-9203(52)90042-4

2. Geser A, Henderson BE, Christensen S. A multipurpose serological survey in Kenya. 2. Results of arbovirus serological tests. Bull World Health Organ. 1970;43:539-52.

3. Chepkorir E, Tchouassi DP, Konongoi SL, Lutomiah J, Tigoi C, Irura Z, et al. Serological evidence of flavivirus circulation in human populations in northern Kenya: an assessment of disease risk 2016-2017. Virol J. 2019;16:65. http:/ / dx.doi.org/10.1186/s12985-019-1176-y

4. Kisuya B, Masika MM, Bahizire E, Oyugi JO. Seroprevalence of Zika virus in selected regions in Kenya. Trans R Soc Trop Med Hyg. 2019;113:735-9. http://dx.doi.org/10.1093/ trstmh/trz077

5. de Oliveira WK, de França GVA, Carmo EH, Duncan BB, de Souza Kuchenbecker R, Schmidt MI. Infection-related microcephaly after the 2015 and 2016 Zika virus outbreaks in Brazil: a surveillance-based analysis. Lancet. 2017;390:861-70. http:/ / dx.doi.org/10.1016/S0140-6736(17)31368-5

6. Hill SC, Vasconcelos J, Neto Z, Jandondo D, Zé-Zé L, Aguiar RS, et al. Emergence of the Asian lineage of Zika virus in Angola: an outbreak investigation. Lancet Infect Dis. 2019;19:1138-47. http:/ / dx.doi.org/10.1016/ S1473-3099(19)30293-2

7. Sheridan MA, Yunusov D, Balaraman V, Alexenko AP, Yabe S, Verjovski-Almeida S, et al. Vulnerability of primitive human placental trophoblast to Zika virus. Proc Natl Acad Sci U S A. 2017;114:E1587-96. http://dx.doi.org/10.1073/ pnas.1616097114

8. Ellis EM, Neatherlin JC, Delorey M, Ochieng M, Mohamed AH, Mogeni DO, et al. A household serosurvey to estimate the magnitude of a dengue outbreak in Mombasa, Kenya, 2013. PLoS Negl Trop Dis. 2015;9:e0003733. http:/ / dx.doi.org/10.1371/journal.pntd.0003733 
9. Johnson BW, Kosoy O, Hunsperger E, Beltran M, Delorey M, Guirakhoo F, et al. Evaluation of chimeric Japanese encephalitis and dengue viruses for use in diagnostic plaque reduction neutralization tests. Clin Vaccine Immunol. 2009;16:1052-9. http:/ / dx.doi.org/10.1128/CVI.00095-09

10. Inwani I, Osoro E, Mugo C, Hunsperger E, Omballa V, Wamalwa $\mathrm{D}$, et al. Zika infection among pregnant women in Mombasa, Coastal Kenya, 2017-2018: preliminary results of a cohort study. Poster presented at: American Society for Tropical Medicine and Hygiene 67th annual meeting; 2018 Oct 30; New Orleans, LA, USA.

Address for correspondence: Marc-Alain Widdowson, Institute of Tropical Medicine, Kronenburgstraat 43, 2000 Antwerp, Belgium; email: mawiddowson@itg.be; Elizabeth Hunsperger, Centers for Disease Control and Prevention, Unit 8900 Box 3600, DPO, AE 09831, USA; email: enh4@cdc.gov

\section{Mycobacterium bovis Pulmonary Tuberculosis after Ritual Sheep Sacrifice in Tunisia}

\author{
Jamal Saad, Sophie Baron, Jean-Christophe Lagier, \\ Michel Drancourt, Phillipe Gautret
}

Author affiliations: IHU Méditerranée Infection, Marseille, France (J. Saad); Aix-Marseille-Université, Marseille (J. Saad, S. Baron, J.-C. Lagier, M. Drancourt, P. Gautret)

DOI: https://doi.org/10.3201/eid2607.191597

A woman in France was diagnosed with pulmonary tuberculosis caused by Mycobacterium bovis after a ritual sheep sacrifice in her home country of Tunisia. This investigation sheds light on ritual sacrifice of sheep as a circumstance in which religious tradition and practices can expose millions of Muslims worldwide to this disease.

ycobacterium bovis is historically responsible for
zoonotic, deadly tuberculosis and has seem-
ingly reemerged in countries where it had previ-
ously vanished following eradication programs in
cattle and the pasteurization of dairy products (1-3).
In most cases, $M$. bovis tuberculosis results from con-
suming unpasteurized milk; extrapulmonary disease is thus the most frequent clinical manifestation (4-6). Also, $M$. bovis could be an airborne zoonotic pathogen causing pulmonary tuberculosis (7). In western Europe countries, most of $M$. bovis human tuberculosis cases are seen in migrants and are associated with travel to the country of origin $(4,5,8,9)$. M. bovis tuberculosis is traced to animal sources, yet reporting of clinical signs and symptoms is often delayed (3). The observation of a woman affected by $M$. bovis tuberculosis who participated in a precisely dated religious practice involving sheep slaughtering provided an opportunity to shed light on these medical aspects.

A 43-year-old unemployed woman born in Tunisia emigrated to France in 2000, married, and had 2 children. The patient had no underlying chronic condition, no medical history, no treatment, no history of smoking, and no toxic habits. She had received the bacillus Calmette-Guérin vaccine during childhood. Her last trip to Tunis and surrounding areas was during July 10-August 28, 2018. The patient denied any contacts with ill persons during her stay. She participated in the Aid-el-Kebir (the Great Festival) Muslim festivities on August 22-23, 2018. After the ritual, her husband slaughtered a veterinary-uncontrolled sheep outside the house by cutting through its neck in an open place and then insufflating air beneath the skin of the dead animal using bellows before butchering the viscera, including lungs, heart, liver, and kidneys, which were put into a container while the digestive tract was put separately into another container. His wife then washed the lungs and the other viscera for $\approx 2$ hours in a confined kitchen, cooked them, and consumed them with her family; she did not experience any injury while butchering the animal.

After her return to France, the patient was apyretic with productive cough, fatigue, and anorexia, which started exactly 22 days after the Aid-el-Kebir festivities ended. The patient attributed symptoms to allergy and did not consult with a healthcare professional. In December 2018, her respiratory tract symptoms persisted; she also developed fatigue, fever, and night sweats and consulted a general practitioner. In January 2019, a computerized tomodensitometry scan confirmed an abscess in the inferior lobe of the patient's left lung with thick walls and an infiltrate in the lower lobe of the right lung. The patient reported fever, cough, expectoration, and anorexia, and lost 5 $\mathrm{kg}$ within 1 month (body mass index 15); we found crackles in both the left inferior and right superior lobes. She did not report hemoptysis, and her physical examination was otherwise unremarkable. Blood examination showed an iron deficiency in microcytic anemia, and her leukocyte count was normal. 